\title{
Candida auris in Healthcare Facilities, New York, USA, 2013-2017
}

\author{
Eleanor Adams, Monica Quinn, Sharon Tsay, Eugenie Poirot, Sudha Chaturvedi, Karen Southwick, \\ Jane Greenko, Rafael Fernandez, Alex Kallen, Snigdha Vallabhaneni, Valerie Haley, Brad Hutton, \\ Debra Blog, Emily Lutterloh, Howard Zucker; Candida auris Investigation Workgroup ${ }^{1}$
}

Candida auris is an emerging yeast that causes healthcareassociated infections. It can be misidentified by laboratories and often is resistant to antifungal medications. We describe an outbreak of $C$. auris infections in healthcare facilities in New York City, New York, USA. The investigation included laboratory surveillance, record reviews, site visits, contact tracing with cultures, and environmental sampling. We identified 51 clinical case-patients and 61 screening case-patients. Epidemiologic links indicated a large, interconnected web of affected healthcare facilities throughout New York City. Of the 51 clinical case-patients, 23 (45\%) died within 90 days and isolates were resistant to fluconazole for 50 (98\%). Of screening cultures performed for 572 persons $(1,136$ total cultures), results were $C$. auris positive for $61(11 \%)$ persons. Environmental cultures were positive for samples from 15 of 20 facilities. Colonization was frequently identified during contact investigations; environmental contamination was also common.

C andida auris is an emerging yeast that has caused healthcare-associated infections on multiple continents (1-13). The organism was first described in 2009 by Satoh et al. for a patient in Japan (14). In November 2016, Vallabhaneni et al. (11) reported cases in the United States. Identification of $C$. auris requires specialized laboratory techniques (15-17). It is often resistant to antifungal

Author affiliations: New York State Department of Health, New Rochelle, New York, USA (E. Adams, K. Southwick); New York State Department of Health, Albany, New York, USA (M. Quinn, S. Chaturvedi, V. Haley, B. Hutton, D. Blog, E. Lutterloh,

H. Zucker); Centers for Disease Control and Prevention, Atlanta, Georgia, USA (S. Tsay, E. Poirot, A. Kallen, S. Vallabhaneni); New York City Department of Health and Mental Hygiene, New York, New York, USA (E. Poirot); New York State Department of Health, Central Islip, New York, USA (J. Greenko); New York State Department of Health, New York (R. Fernandez); State University of New York at Albany School of Public Health, Albany, New York, USA (V. Haley, D. Blog, E. Lutterloh)

DOI: https://doi.org/10.3201/eid2410.180649 medications (18), causes invasive infections $(1,4,5)$ and outbreaks $(8,10)$, and has become endemic to hospitals in some parts of the world $(2,5,6)$. Therefore, its detection in New York, USA, healthcare facilities is concerning. We describe an ongoing outbreak of healthcare-associated $C$. auris cases involving multiple healthcare facilities in New York City (NYC), New York, USA, during 2013-2017.

\section{Methods}

\section{Definitions and Data Analysis}

We defined a case-patient as a person for whom a culture was positive for $C$. auris. Clinical cases are those for which the culture was obtained to diagnose or treat disease; screening cases are those for which the culture was obtained for surveillance purposes. We defined contacts as persons who had an epidemiologic link to a case-patient in place or time. We included clinical cases reported by April 30, 2017. Because surveillance cultures of contacts are performed after an associated clinical case is reported, we included surveillance cultures that were collected by June 26, 2017, and had final results available by July 19, 2017, which enabled us to better approximate the number of screening cases associated with clinical cases described herein. Data were analyzed by using SAS 9.4 (SAS Institute, Cary, NC, USA) and Excel 2016 (Microsoft, Redmond, WA, USA).

\section{Case Finding and Investigation}

In June 2016, the Centers for Disease Control and Prevention (CDC) issued an alert about C. auris (19), after which the New York State Department of Health (NYS$\mathrm{DOH}$ ) issued an advisory (20) to inform healthcare facilities about the emerging pathogen and request that they notify NYSDOH of potential cases and forward suspected isolates to the New York state public health laboratory (Mycology Laboratory at Wadsworth Center, Albany, NY, USA). In November 2016, NYSDOH issued a

${ }^{1}$ Additional members of the workgroup are listed at the end of this article. 
follow-up advisory (21) requesting that laboratories query their records for isolates of $C$. auris or species that could be confused with $C$. auris, such as $C$. haemulonii. Cases were also identified through active surveillance methods, including direct outreach to healthcare facilities and laboratories. Each clinical case was investigated through medical record reviews, contact tracing, and screening of contacts for colonization.

\section{Contact Tracing}

We obtained the names of persons who had resided in the same room as a case-patient in the 90 days before diagnosis for the case-patient. When close contacts could be located, we attempted to obtain samples for culture.

\section{Surveillance and Infection Control Assessments}

To emphasize the importance of detection, assist with infection control efforts, and conduct point prevalence surveys of facility contacts, we conducted site visits to facilities where transmission was suspected; our analysis included visits made through June 26, 2017. Initial point prevalence surveys included only a composite swab sample from the axilla and groin; subsequent surveys added a swab sample from the nares. For some persons, swab samples for surveillance cultures to identify persistent colonization were obtained and included samples from the axilla, groin, nares, rectum, wounds, and sites of noninvasive clinical infection. We also assessed key areas of healthcare infection control, including administrative support, hand hygiene, standard and transmission-based precautions, and environmental cleaning; we followed up with detailed assessments in specific areas as needed.

\section{Environmental Investigation}

Whenever possible, we obtained samples from the environments of facilities where case-patients were admitted or resided. We concentrated on surfaces that were frequently touched and on objects in case-patients' rooms.

\section{Laboratory Techniques}

To isolate $C$. auris from patient screening swab and environmental specimens, we used the method described by Welsh et al., with slight modification (15). In brief, we used the ESwab Culture and Transport system (Becton Dickinson, Franklin Lakes, NJ, USA) and placed the samples in $1 \mathrm{~mL}$ liquid Amies transport medium. Samples were vortexed for $30 \mathrm{~s}$, after which $50 \mu \mathrm{L}$ was plated on nonselective Sabouraud dextrose agar containing antibacterials (SDA-A), $50 \mu \mathrm{L}$ was plated on selective media including SDA-A enriched with $10 \%$ salt (SDA-AS), and $200 \mu \mathrm{L}$ was transferred to $5 \mathrm{~mL}$ of Sabouraud dextrose broth containing antibacterials and $10 \%$ salt (SDB-AS). Later, for more selective recovery of $C$. auris from surveillance samples, we placed dulcitol, instead of dextrose, in the selective enrichment media.

We collected environmental samples by using sponge sticks (3M Health Care, St. Paul, MN, USA) and placed them in a zip-top bag containing $45 \mathrm{~mL}$ of phosphatebuffered saline (PBS) with $0.02 \%$ Tween 80 . The bags were gently shaken for $1 \mathrm{~min}$ at $260 \mathrm{rpm}$ in a Stomacher 400 Circulator (Laboratory Supply Network, Inc., Atkinson, NH, USA). The suspension without the sponge was poured in a $50-\mathrm{mL}$ conical tube and centrifuged at 4,000 $\mathrm{rpm}$ for $5 \mathrm{~min}$; supernatant was then decanted, leaving $\approx 3$ $\mathrm{mL}$ of liquid in the bottom of the tube. We placed $50 \mu \mathrm{L}$ of sponge suspension on different agar media and placed $1 \mathrm{~mL}$ of sponge suspension in $5 \mathrm{~mL}$ of SDB-AS broth, as we had done for patient swab samples.

Agar plates and broth tubes were incubated at $40^{\circ} \mathrm{C}$ for at least 2 weeks. To check for purity, we first streaked recovered yeast isolates on CHROMagar Candida medium (Difco; Becton Dickinson, Baltimore, MD, USA) and then subcultured them on SDA overnight and processed them for identification by matrix-assisted laser desorption/ionization time-of-flight (MALDI-TOF) mass spectrometry by using the standard ethanol-formic acid extraction procedure (22). Spectra were analyzed by using Flex Control 3.1 software (Bruker Daltonics, Inc., Billerica, MA, USA) and MALDI Biotyper OC version 3.1 (Bruker Daltonics, Bremen, Germany); per manufacturer's instructions, a score of $\geq 2.0$ was used to identify Candida to the species level. The in-house MALDI-TOF database was enriched by adding spectra from several $C$. auris isolates from the current outbreak and by adding reference isolates from the CDC AR bank (https://www.cdc.gov/drugresistance/resistancebank/index.html); their identity was confirmed by DNA sequencing. To check for purity, we streaked the clinical isolates of yeasts received from healthcare facilities onto CHROMagar Candida medium and used MALDI-TOF mass spectrometry for identification as described.

The MICs of azoles and echinocandins were determined by using broth microdilution with custom TREK frozen broth microdilution panels (catalog no. CML2FCAN; Thermo Fisher Scientific, Marietta, OH, USA) (23). In brief, we prepared a suspension of $C$. auris at a concentration of $0.5 \times 10^{3}$ to $2.5 \times 10^{3}$ in RPMI-1640 medium (with glutamic acid and phenol red, and without bicarbonate; Sigma-Aldrich, St. Louis, MO, USA) and $0.2 \%$ glucose buffered to $\mathrm{pH} 7$ with $0.165 \mathrm{~mol} / \mathrm{L} 3-\mathrm{N}$ morpholinepropanesulfonic acid (Sigma-Aldrich). We dispensed $100 \mu \mathrm{L}$ C. auris inoculum into each well of the TREK plate. MICs of amphotericin B and 5-flucytosine were determined by Etest as recommended by the manufacturer (AB Biodisk; bioMérieux, Solna, Sweden) except that MICs were read at $24 \mathrm{~h}$ after incubation or until a confluent lawn of growth was seen. For Etests, the yeast inoculum was streaked on 
RPMI medium containing 2\% glucose and $1.5 \%$ agar, and then E-test strips were applied. C. krusei (ATCC 6258) and C. parapsilosis (ATCC 22019) were used as quality control strains. The TREK broth and Etest plates were incubated at $35^{\circ} \mathrm{C}$ and read visually after 24 hours. Because there are no established C. auris-specific susceptibility breakpoints, we used tentative breakpoints published by CDC (16).

Environmental surveillance samples (sponges) were also processed by real-time PCR (24). In brief, $1 \mathrm{~mL}$ of sponge liquid was washed twice with PBS containing $0.1 \%$ bovine serum albumin; as an inhibition control, pellet was resuspended in $50 \mu \mathrm{L}$ PBS with $0.1 \%$ bovine serum albumin containing bicoid plasmid. Each sample went through freezing, heating, bead-beating, and centrifugation, and $5 \mu \mathrm{L}$ of extracted DNA was tested in duplicate by real-time PCR. According to receiver operator characteristic curve analysis, a cycle threshold value of $\leq 38$ was reported as positive and $>38$ was reported as negative. If PCR inhibition was observed, specimens were reported as inconclusive.

\section{Results}

\section{Epidemiologic Investigation}

We detected 51 clinical and 61 screening cases (Figure 1). All but 1 of the clinical cases from New York were diagnosed in NYC: 21 from 7 hospitals in Brooklyn, 16 from 3 hospitals and 1 private medical office in Queens, 12 from 5 hospitals and 1 long-term acute care hospital in Manhattan, and 1 from a hospital in the Bronx. One clinical case was identified in a western New York hospital in a patient who had recently been admitted to an involved Brooklyn hospital. Of the 51 clinical case-patients, 31 (61\%) had resided in long-term care facilities (LTCFs) immediately before being admitted to the hospital in which their infection was diagnosed, and 19 of these 31 resided in skilled nursing facilities with ventilator beds (VSNFs); 1 (2\%) resided in a long-term acute care hospital; $5(10 \%)$ had been transferred from another hospital; and $4(8 \%)$ had traveled internationally within 5 years before diagnosis.

Exploration of epidemiologic links revealed a large, interconnected web of affected healthcare facilities throughout NYC (Figure 2). Determining the facility of acquisition of $C$. auris infection or colonization was difficult or impossible because of multiple healthcare exposures and because the incubation period is unknown.

\section{Clinical Characteristics}

The median age of clinical case-patients was 72 years (range 21-96 years); 26 (51\%) were male. All patients had serious concurrent medical conditions; a substantial proportion required mechanical ventilation or central venous catheters or gastrostomy tubes (Table 1). Initial positive cultures were from blood $(31 / 51,61 \%)$, bile $(3 / 51$, $6 \%)$, urine $(4 / 51,8 \%)$, respiratory specimens $(4 / 51,8 \%)$, wounds $(3 / 51,6 \%)$, catheter tips $(2 / 51,4 \%)$, and 1 each from bone, ear, jejunal biopsy sample, and skin. The 30day mortality rate was $14 / 51(27 \%)$, and the 90 -day rate was $23 / 51(45 \%)$. For those with initial isolates from blood, the 30-day mortality rate was $12 / 31(39 \%)$ and the 90 -day rate was $18 / 31(58 \%)$. The number of deaths attributable to C. auris infection is unknown.

\section{Surveillance Cultures}

As part of point prevalence surveys and contact investigations, we performed 1,136 screening cultures for $C$. auris colonization for 572 persons not known to be infected and who resided in or were admitted to 19 facilities (9 hospitals; 10 LTCFs, of which 7 were VSNFs), 4 healthcare workers, and 4 family members of 1 clinical case-patient. At least 1 culture was positive for $C$. auris for $61(11 \%)$ persons at $12(60 \%)$ facilities (5 hospitals and 7 LTCFs [including 5

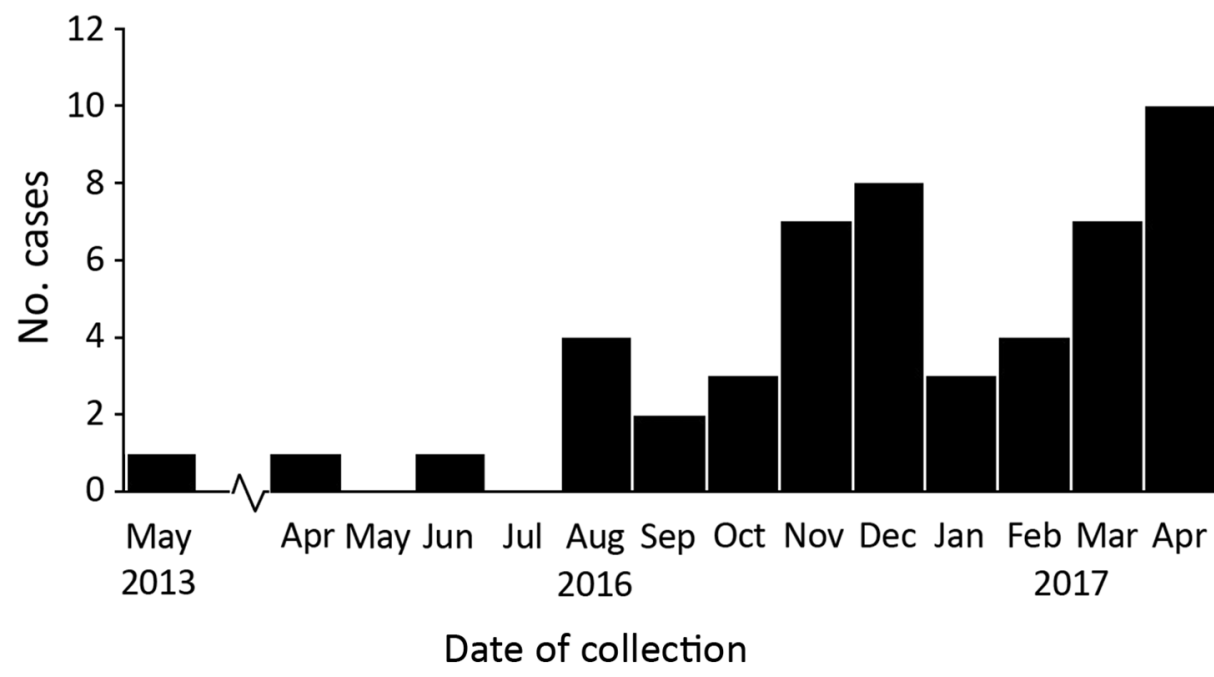

Figure 1. Number of confirmed clinical cases of Candida auris in New York, USA, May 2013-April 2017. Dates indicate the month that the first sample positive for $C$. auris was collected. The cases from May 2013, April 2016, and June 2016 were retrospectively identified after the June 2016 clinical alert from the Centers for Disease Control and Prevention was issued (19). The case from 2013, in a patient who had traveled to New York City from abroad for medical care, was probably a distinct importation with no further spread. 
Figure 2. Epidemiologic links between healthcare facilities affected by Candida auris, New York, USA, 2013-2017. Arrows between facilities denote transfer of case-patients from one facility to the other within 90 days before collection date of first positive culture. Bold arrows indicate transfer of $>1$ case-patient. Bold boxes indicate hospitals; nonbold boxes indicate long-term care facilities; boxes with roofs indicate private residences. Numbers indicate numbers of clinical cases $(\mathrm{C})$ and screening cases (S) at that facility. Screening cases are placed at the facility of diagnosis. Clinical cases are also shown at the facility of diagnosis unless the specimen was collected during the first week of admission at the diagnosing facility; in such situations, the cases are shown at the previous facility.

VSNFs]) and 1 family caregiver at a private residence. At the time of sample collection, $19(31 \%)$ of these 61 persons were admitted to hospitals and $42(67 \%)$ resided at LTCFs (40 [66\%] at VSNFs). Culture results were positive for $13 \%$ of those who were tested while living at LTCFs and $8 \%$ of those in hospitals.

For 38 persons (clinical and screening case-patients), follow-up cultures were performed, either for clinical reasons or to determine whether they remained colonized. Long-term colonization was common (Figure 3).

Of 346 persons for whom nares and composite axilla-groin samples for culture were collected on the same day, results were positive for at least 1 site for $36(10 \%)$.
Of these 36, results were positive for both sites for 14 $(39 \%)$, at axilla-groin only for $13(36 \%)$, and at nares only for $9(25 \%)$.

\section{Environmental Cultures and PCR}

Of 781 environmental samples from 20 facilities (12 hospitals and 8 LTCFs [5 VSNFs]), 62 (8\%) from 15 facilities (9 hospitals and 6 LTCFs [4 VSNFs]) were positive for C. auris by culture. In addition, 19 samples from 4 facilities were positive by PCR; culture results for 3 of these 4 facilities were also positive. Contamination of surfaces and objects in case-patients' rooms and mobile equipment outside the rooms was common (Table 2). High-yield items included

\begin{tabular}{lc}
\hline Table 1. Selected concurrent medical conditions and medical interventions for 51 persons with Candida auris infection, New York, \\
USA, 2013-2017 & No. (\%) persons \\
\hline Characteristic & $33(65)$ \\
\hline Concurrent condition & $17(33)$ \\
Respiratory insufficiency requiring support & $24(47)$ \\
Mechanical ventilation at time of diagnosis & $18(35)$ \\
Neurologic disease* & $11(22)$ \\
Diabetes & $5(10)$ \\
Malignancies & $8(16)$ \\
Colon cancer & $7(14)$ \\
End-stage renal disease & $1(2)$ \\
Hemodialysis & $10(20)$ \\
Kidney transplant & $2(4)$ \\
Decubitus ulcers & \\
Otitis with complications & $7(14)$ \\
\hline Medical interventions & $31(61)$ \\
Hemodialysis & $27(53)$ \\
Central venous catheter within 7 d before first positive culture for C. auris & $25(49)$ \\
Gastrostomy tube at time of diagnosis & $42(82)$ \\
Receipt of systemic antifungal medication within 90 d before first culture positive for C. auris & \\
Receipt of systemic antibiotics within 14 d before first culture positive for C. auris & \\
\hline
\end{tabular}




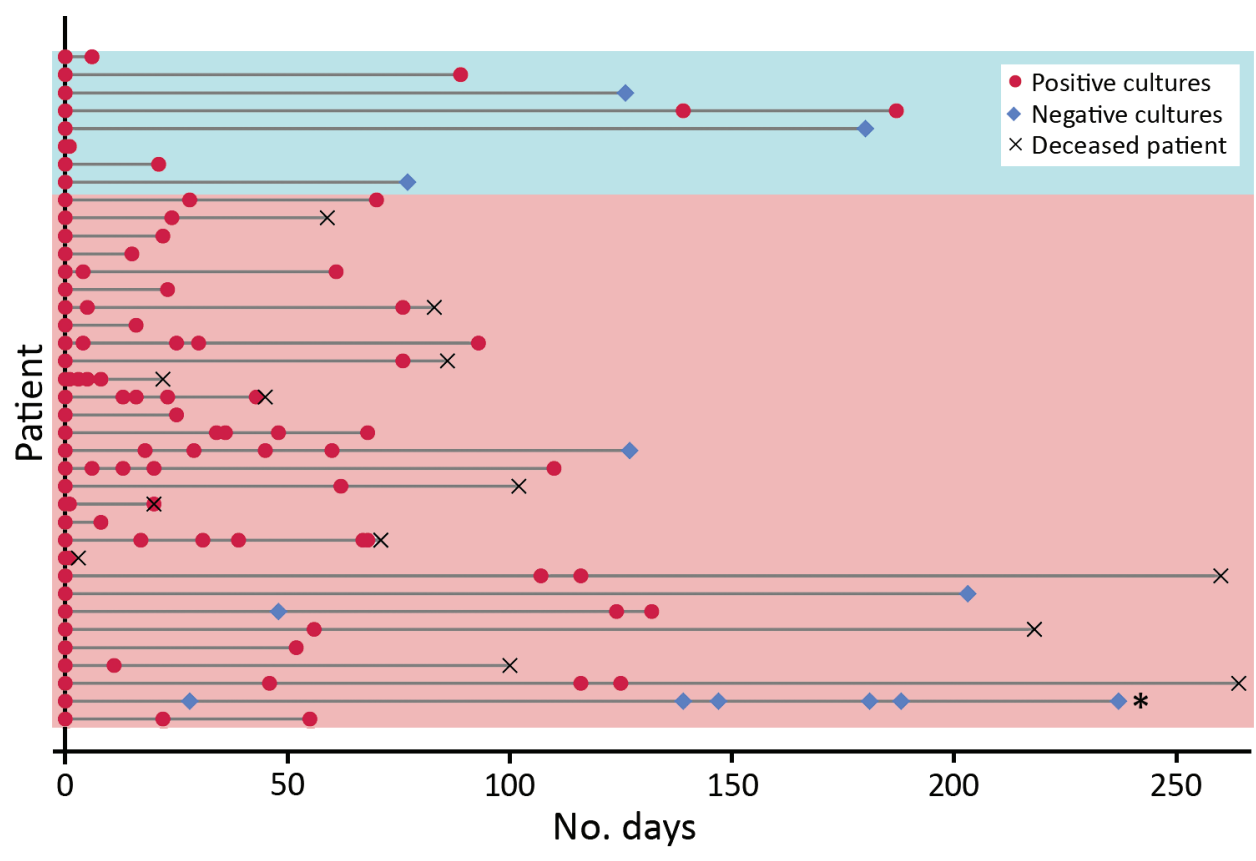

Figure 3. Long-term Candida auris colonization of clinical and screening case-patients, New York, USA, 2013-2017. Each patient for whom followup cultures were performed is represented by a horizontal line. The bottom 30 lines (pink shading) indicate clinical casepatients; the top 8 (blue shading) indicate screening casepatients. Follow-up cultures were collected from a variety of sites, typically axilla and groin and often nares, rectum, urine, and wounds. Persons were considered free of colonization with $\mathrm{C}$. auris and eligible for removal of contact precautions when 2 sets of surveillance cultures at multiple sites, taken at least 1 week apart, were negative; only 1 person indicated on the figure (second from bottom) met this criterion. bedrails, IV poles, beds, privacy and window curtains, windows, and floors.

\section{Laboratory Identification of Clinical Isolates}

From July 2016 through April 30, 2017, NYSDOH received 99 first isolates of a variety of yeasts from 99 persons, which clinical laboratories had sent for testing for $C$. auris (Table 3). Of those, 51 (52\%) isolates were determined to be $C$. auris and represent the 51 clinical cases. Of the 99 isolates, 38 had been initially identified by the clinical laboratory as $C$. haemulonii, but NYSDOH determined 35 of those to be $C$. auris. Of 13 yeasts received with no identification, 11 were determined to be $C$. auris, and of 6 received with a preliminary identification of C. auris, 5 were confirmed as such.

\section{Susceptibility to Antifungal Medications}

Of 51 initial C. auris isolates recovered from clinical casepatients, 50 (98\%) were resistant to fluconazole (Table 4) and $13(25 \%)$ were resistant to fluconazole and amphotericin B. No initial isolates were resistant to echinocandins, although subsequent isolates obtained from 3 persons who had received an echinocandin acquired resistance to it. According to whole-genome sequencing at CDC, 50 (98\%) of 51 isolates belonged to a South Asia clade (25); the other less related isolate was the only isolate susceptible to fluconazole.

\section{Infection Control}

Infection control assessments were conducted at 14 LTCFs and 12 hospitals affected by C. auris. Adherence to recommended infection control practices, such as implementation of contact precautions, varied. Specific observations were made in the areas of hand hygiene, contact precautions, use of personal protective equipment (PPE), and environmental cleaning and disinfection.

Hand hygiene problems included frequent suboptimal availability of alcohol-based hand sanitizers. Sanitizers were completely absent in 1 LTCF.

A common problem with implementation of contact precautions was ineffective signage. One facility had no signs or other effective systems to identify persons around whom contact precautions should be taken. Compliance with signs that consisted only of instructions to see the nurse before entering was poor. In one instance, a physician entered a room with such a sign and provided care without donning PPE; when questioned, he stated, "I don't see an isolation sign."

Problems with PPE use included lack of knowledge about which PPE was indicated, improper donning and doffing (e.g., gowns not covering shoulders or not being tied), and lack of availability of appropriate PPE. In 1 LTCF, PPE was locked in a closet; in another, the PPE carts were empty and staff were unable to locate supplies to replenish them; in a third, aprons were used instead of gowns.

Environmental cleaning and disinfection observations included use of household cleaners instead of Environmental Protection Agency-registered hospital-grade disinfectants (at some LTCFs), use of disinfectants without appropriate label claims, inadequate disinfection of shared equipment, and lack of knowledge of contact times. 
Table 2. Environmental contamination with Candida auris in healthcare facilities, New York, USA, 2013-2017*

\begin{tabular}{|c|c|c|c|c|}
\hline Category, object or surface & No. samples & $\begin{array}{c}\text { Positive by } \\
\text { culture, no. (\%) }\end{array}$ & $\begin{array}{c}\text { Positive by PCR and } \\
\text { negative by culture, } \\
\text { no. (\%) }\end{array}$ & $\begin{array}{c}\text { Negative by } \\
\text { culture and PCR, } \\
\text { no. }(\%)\end{array}$ \\
\hline \multicolumn{5}{|l|}{ Near-patient surfaces and objects in rooms } \\
\hline Bedside/over bed table & 44 & $2(5)$ & $2(5)$ & $40(91)$ \\
\hline Bed rail & 49 & $7(14)$ & $5(10)$ & $37(76)$ \\
\hline TV remote/call button & 36 & $2(6)$ & $2(6)$ & $32(89)$ \\
\hline IV poles & 21 & $5(24)$ & $1(5)$ & $15(71)$ \\
\hline Bed & 17 & $4(24)$ & 0 & $13(77)$ \\
\hline Privacy curtain & 6 & $2(33)$ & 0 & $4(67)$ \\
\hline Miscellaneous other† & 5 & 0 & $1(20)$ & $4(80)$ \\
\hline Total & 178 & $22(12)$ & $11(6)$ & $145(82)$ \\
\hline \multicolumn{5}{|l|}{ Other surfaces and objects in rooms } \\
\hline Door knob/handle & 36 & $1(3)$ & $1(3)$ & $34(94)$ \\
\hline Sink & 27 & $1(4)$ & $2(7)$ & $24(89)$ \\
\hline Window & 22 & $3(14)$ & $1(5)$ & $18(82)$ \\
\hline Floor & 17 & $4(24)$ & 0 & $13(77)$ \\
\hline Furniture & 27 & $3(11)$ & 0 & $24(89)$ \\
\hline Window curtain & 11 & $3(27)$ & 0 & $8(73)$ \\
\hline Light switch & 9 & 0 & 0 & $9(100)$ \\
\hline Closet & 6 & 0 & 0 & $6(100)$ \\
\hline Wall & 4 & $1(25)$ & 0 & $3(75)$ \\
\hline Bathroom & 4 & $1(25)$ & 0 & $3(75)$ \\
\hline Countertop & 4 & $1(25)$ & 0 & $3(75)$ \\
\hline Toilet & 4 & 0 & 0 & $4(100)$ \\
\hline Miscellaneous other & 16 & $2(13)$ & 0 & $14(88)$ \\
\hline Total & 187 & $20(21)$ & $4(2)$ & $163(87)$ \\
\hline \multicolumn{5}{|l|}{ Equipment in room } \\
\hline Ventilator/respiratory equipment & 12 & $1(8)$ & 0 & $11(92)$ \\
\hline Pump & 4 & 0 & 0 & $4(100)$ \\
\hline Miscellaneous other§ & 19 & $4(21)$ & 0 & $15(79)$ \\
\hline Total & 35 & $5(14)$ & 0 & $30(86)$ \\
\hline \multicolumn{5}{|l|}{ Equipment outside of room } \\
\hline Clean supply cart & 51 & $1(2)$ & 0 & $50(98)$ \\
\hline Ventilator/respiratory equipment & 45 & $1(2)$ & 0 & $44(98)$ \\
\hline Vital sign machine & 21 & $3(14)$ & $1(5)$ & $17(81)$ \\
\hline Normothermia system (e.g., Bair hugger) & 20 & $1(5)$ & 0 & $19(95)$ \\
\hline Computer workstation & 20 & 0 & 0 & $20(100)$ \\
\hline Thermometer & 14 & $1(7)$ & $1(7)$ & $12(86)$ \\
\hline PPE/isolation cart/box & 12 & $1(8)$ & $1(8)$ & $10(83)$ \\
\hline Lift/scale & 11 & $2(18)$ & 0 & $9(82)$ \\
\hline Glucometer & 11 & 0 & 0 & $11(100)$ \\
\hline Housekeeping cart & 9 & 0 & $1(11)$ & $8(89)$ \\
\hline Dialysis equipment & 7 & $1(14)$ & 0 & $6(86)$ \\
\hline Suction canister & 6 & $1(17)$ & 0 & $5(83)$ \\
\hline Ultrasonography equipment & 4 & 0 & 0 & $4(100)$ \\
\hline Miscellaneous otherq & 29 & $1(3)$ & 0 & $28(97)$ \\
\hline Total & 260 & $13(5)$ & $4(2)$ & $243(94)$ \\
\hline \multicolumn{5}{|c|}{$\begin{array}{l}\text { *A total of } 660 \text { samples were collected from surfaces, objects, and equipment in the rooms of } C \text {. auris case-patients and from mobile equipment outside } \\
\text { the rooms on the affected nursing units. In addition, } 62 \text { samples from surfaces within the nursing units but outside the patient rooms and } 23 \text { samples from } \\
\text { outside the affected nursing units were negative by culture and PCR. The location of } 36 \text { samples could not be ascertained; } 2 \text { were positive by culture. } \\
\text { PPE, personal protective equipment; TV, television. } \\
\text { †PCR positive from light cord. } \\
\text { tCultures positive from handrail and phone. } \\
\text { §Cultures positive from glucometers }(n=2) \text {, vital signs machine, and stretcher. } \\
\text { TCulture positive from bedpan flusher. }\end{array}$} \\
\hline
\end{tabular}

\section{Discussion}

This large, citywide, multiple-institution outbreak of $C$. auris infections is ongoing. As of February 2018, most confirmed clinical cases in the United States had been identified in New York, and case numbers continue to grow. The reasons for the preponderance of cases in New York are unknown; possibilities include a true higher prevalence from multiple introductions into this international port of entry, more complete detection from aggressive case finding, presence of a large interconnected network of healthcare facilities in NYC, or a combination of all 3 factors.

Transmission is ongoing in healthcare facilities, primarily among patients with extensive healthcare exposures. C. auris has been cultured from rooms and equipment in multiple facilities, and close contacts of case-patients have become colonized. Infection control lapses have probably amplified this process. 
Table 3. Isolates received by the New York State public health laboratory, Wadsworth Center, Albany, NY, USA, from clinical laboratories for the purpose of identifying or excluding Candida auris, through April 30, 2017*

\begin{tabular}{|c|c|c|c|c|c|}
\hline \multirow[b]{2}{*}{ Organism, no. isolated } & \multicolumn{4}{|c|}{ Clinical laboratories' identification system } & \multirow{2}{*}{$\begin{array}{c}\text { Wadsworth Center identification } \\
\text { using MALDI-TOF MS, no. isolates } \neq\end{array}$} \\
\hline & $\mathrm{API}$ & VITEK 2 & VITEK MS† & Other & \\
\hline \multirow[t]{2}{*}{ Candida haemulonii, 38} & & 36 & & $1 \S$ & C. auris, 35 \\
\hline & & & & $1 \rrbracket$ & $\begin{array}{c}\text { C. haemulonii, 1; Candida } \\
\text { duobushaemulonii, 1; Candida } \\
\text { glabrata,1 }\end{array}$ \\
\hline No identification, 13 & 2 & 2 & 9 & & $\begin{array}{c}\text { C. auris, } 11 \\
\text { C. glabrata, } 2\end{array}$ \\
\hline C. auris, 6 & & & & $6 \S$ & $\begin{array}{c}\text { C. auris, } 5 \\
\text { C. duobushaemulonii, } 1\end{array}$ \\
\hline Candida famata, 5 & 1 & 3 & & 1\# & $\begin{array}{c}\text { C. guilliermondii, 1; C. Iusitaniae, } 1 \text {; } \\
\text { Candida parapsilosis, 2; Candida } \\
\text { fermentati, } 1\end{array}$ \\
\hline Candida glabrata, 1 & & 1 & & & C. glabrata, 1 \\
\hline Candida guilliermondii, 1 & & 1 & & & C. guilliermondii, 1 \\
\hline Candida lusitaniae, 1 & & 1 & & & C. Iusitaniae, 1 \\
\hline Candida sphaerica, 1 & & 1 & & & Saccharomyces cerevisiae, 1 \\
\hline Cryptococcus laurentii, 1 & & 1 & & & Cryptococcus neoformans, 1 \\
\hline C. neoformans, 1 & & 1 & & & S. cerevisiae, 1 \\
\hline C. famata/C. guilliermondii, 1 & & 1 & & & C. parapsilosis, 1 \\
\hline C. famata/C. parapsilosis, 1 & & 1 & & & C. parapsilosis, 1 \\
\hline $\begin{array}{l}\text { C. famata/C. parapsilosis/ } \\
\text { Candida tropicalis, } 1\end{array}$ & & 1 & & & C. parapsilosis, 1 \\
\hline Candida dubliniensis/C. haemulonii, 1 & & 1 & & & C. glabrata, 1 \\
\hline C. Iusitaniae/Candida utiliz, 1 & & & 1 & & C. Iusitaniae, 1 \\
\hline $\begin{array}{l}\text { Candia sphaerica/Rhodotorula glutinis/ } \\
\text { Rhodotorula mucilaginosa/C. laurentii, } 1\end{array}$ & & 1 & & & C. parapsilosis, 1 \\
\hline $\begin{array}{l}\text { Zygosaccharomyces bailii/C. sake/C. } \\
\text { famata/Candida lipolytica, } 1\end{array}$ & & 1 & & & C. glabrata, 1 \\
\hline S. cerevisiae, 23 & 7 & 16 & & & S. cerevisiae, 23 \\
\hline Trichosporon mucoides, 1 & & 1 & & & T. mucoides, 1 \\
\hline $\begin{array}{l}\text { *API, bioMérieux, Inc., Durham, NC, USA; MAL } \\
\text { MS, bioMérieux, Solna, Sweden. MALDI-TOF, } \\
\text { †No C. auris entry. } \\
\text { †Research use only library was expanded in-ho } \\
\text { https://www.cdc.gov/drugresistance/resistance- } \\
\text { §MALDI-TOF MS, research use only library wit } \\
\text { TBD Phoenix Automated Microbiology System } \\
\text { \#RapID YEAST PLUS System (Thermo Fisher }\end{array}$ & $\begin{array}{l}\text { S, Bru } \\
\text { isted le } \\
\text { ding } 1 \\
\text { x.html) } \\
\text { s entrie } \\
\text { ostics, } \\
\text { Walthe }\end{array}$ & $\begin{array}{l}\text { altonics, Inc } \\
\text { esorption/ic } \\
\text { uris isolate } \\
\text { C. auris is } \\
\text { KS, MD, US } \\
\text { A, USA). }\end{array}$ & $\begin{array}{l}\text { Billerica, MA, US } \\
\text { zation time-of-flic } \\
\text { omprising clade } \\
\text { ates from New } Y\end{array}$ & $\begin{array}{l}; \text { VITEK } \\
\text { t: MS, } n \\
\text { I-IV (CD } \\
\text { k in } 201\end{array}$ & $\begin{array}{l}\text {, bioMérieux, Durham, NC, USA; VITEK } \\
\text { ss spectrometry. } \\
\text {-AR bank, } \\
\text { comprising clades I and II. }\end{array}$ \\
\hline
\end{tabular}

Factors that contribute to transmission may include prolonged colonization of clinical and screening case-patients and environmental contamination. Persistent colonization of affected persons and the lack of an accepted decolonization regimen result in a large reservoir of persons carrying the organism. As Welsh et al. (15) demonstrated, $C$. auris can remain viable on inanimate surfaces for long periods, necessitating scrupulous environmental cleaning and disinfection. Affected patients frequently have extensive contact with multiple healthcare facilities, highlighting the value of careful and thorough communication at transfer.

The clinical cases in the New York outbreak are similar to clinical cases described elsewhere. Fungemia is a commonly reported clinical infection; $76 \%$ of infections reported in a series from Colombia (9) and $58 \%$ in a series from India (4) were bloodstream infections. These percentages are comparable to the findings from this New York series in which $61 \%$ of initial clinical isolates were from blood. Among medically fragile patients in NYC who had a history of extensive contact with healthcare facilities, clinicians should include $C$. auris in the differential diagnosis for patients with symptoms compatible with bloodstream infection.

Limitations of this investigation include the inability to determine where $C$. auris was acquired for most cases because of multiple healthcare exposures. Point prevalence surveys have not yet been conducted at all involved facilities. The best uses for and interpretation of PCR results are still being determined, especially when PCR is positive but culture result is negative. This investigation did not assess transmission in the community or outpatient settings; other investigators have described $C$. auris infections associated with an outpatient clinic (12).

Given the consequences of the development and spread of multidrug-resistant bacteria over the past several decades, the prospect of an endemic or epidemic multidrug-resistant yeast in US healthcare facilities is troubling. Data from other countries show that $C$. auris can become established within facilities. Chowdhary et al. 
Table 4. Antifungal susceptibility data for first Candida auris isolates from 51 clinical cases, New York, USA, 2013-2017

\begin{tabular}{|c|c|c|c|c|}
\hline Antifungal & $\begin{array}{c}\text { Tentative resistance } \\
\text { breakpoint (16) }\end{array}$ & $\mathrm{MIC}_{50} \dagger$ & MIC range† & No. (\%) resistant \\
\hline Fluconazole & $\geq 32$ & $>256$ & 8.00 to $\geq 256$ & $50(98)$ \\
\hline Itraconazole & $\bar{N} A$ & 0.500 & $0.25-1.00$ & NA \\
\hline Voriconazole & NA & 2.000 & $0.50-4.00$ & NA \\
\hline Posaconazole & NA & 0.250 & $0.12-0.50$ & NA \\
\hline Isavuconazole & NA & 0.500 & $0.25-2.00$ & NA \\
\hline Caspofungin & $\geq 2$ & 0.060 & $0.03-0.25$ & 0 \\
\hline Micafungin & $>4$ & 0.120 & $0.06-0.25$ & 0 \\
\hline Anidulafungin & $>4$ & 0.250 & $0.12-0.50$ & 0 \\
\hline Amphotericin B & $>2$ & 1.500 & $0.50-4.00$ & $15(29)$ \\
\hline Flucytosine & $\overline{\mathrm{N}} \mathrm{A}$ & 0.125 & $0.125-0.25$ & NA \\
\hline
\end{tabular}

${ }^{*}$ NA, not available.

†MICs for azoles and echinocandins are defined as the lowest drug concentration that caused $50 \%$ growth inhibition compared with the drug-free controls; MICs for amphotericin B and flucytosine are defined as the lowest concentration at which there was $100 \%$ growth inhibition. MIC 50 was defined as the MIC at which $\geq 50 \%$ of the isolates of $C$. auris tested were inhibited.

(2) report that $C$. auris accounted for $5 \%$ of candidemia cases in a pediatric hospital and $30 \%$ in a tertiary general hospital in India. Chakrabarti et al. (6) report that $C$. auris was isolated from 19 of 27 intensive care units throughout India and accounted for $5.2 \%$ of Candida isolates from intensive care units. Okinda et al. (26) report that $38 \%$ of candidemia cases at a referral hospital in Africa were caused by $C$. auris, surpassing $C$. albicans cases $(27 \%)$. Schelenz et al. (10) describe an outbreak in a London hospital that affected 50 patients.

Infection control lapses observed during site visits prompted NYSDOH leadership to conduct educational webinars for New York state clinicians and onsite infection control-focused reviews of all nonfederal hospitals and LTCFs in Brooklyn and Queens. NYSDOH also created a web page for healthcare personnel and the public (27). Intensive infection prevention and control efforts continue; the goals are delaying endemicity, preventing outbreaks within facilities, reducing transmission and geographic spread, and blunting the effect of $C$. auris in New York and the rest of the United States.

Additional members of the Candida auris Investigation Workgroup: Coralie Bucher, Richard L. Erazo, Rosalie Giardina, Janet Glowicz, Brendan R. Jackson, Ronald Jean Denis, Jillian Karr, Gale Liddell, Anastasia Litvintseva, Shawn R. Lockhart, Abimbola Ogundimu, Rutvik Patel, Maroya Walters, Rory Welsh, and YanChun Zhu.

\section{Acknowledgments}

We thank Karlyn Beer, Elizabeth Berkow, Nancy Chow, Kaitlin Forsberg, Lalitha Gade, Lynn Leach, Brittany O'Brien, Stephanie Ostrowski, Kate Russell, Alicia Shugart, and Nimalie Stone for their contributions to this investigation.

This work was supported in part by the Epidemiology and Laboratory Capacity for Infectious Diseases Cooperative Agreement nos. NU50CK000423 and CK000423-01S2, funded by CDC.

\section{About the Author}

Dr. Adams is a public health physician with the NYSDOH, Metropolitan Area Regional Office, where she serves as the supervisor of the Healthcare Epidemiology and Infection Control Program, overseeing outbreak investigations in healthcare facilities in NYC, Long Island, and the Lower Hudson Valley.

\section{References}

1. Lee WG, Shin JH, Uh Y, Kang MG, Kim SH, Park KH, et al. First three reported cases of nosocomial fungemia caused by Candida auris. J Clin Microbiol. 2011;49:3139-42. http://dx.doi.org/10.1128/JCM.00319-11

2. Chowdhary A, Sharma C, Duggal S, Agarwal K, Prakash A, Singh PK, et al. New clonal strain of Candida auris, Delhi, India. Emerg Infect Dis. 2013;19:1670-3. http://dx.doi.org/10.3201/ eid1910.130393

3. Sarma S, Kumar N, Sharma S, Govil D, Ali T, Mehta Y, et al. Candidemia caused by amphotericin B and fluconazole resistant Candida auris. Indian J Med Microbiol. 2013;31:90-1. http://dx.doi.org/10.4103/0255-0857.108746

4. Chowdhary A, Anil Kumar V, Sharma C, Prakash A, Agarwal K, Babu R, et al. Multidrug-resistant endemic clonal strain of Candida auris in India. Eur J Clin Microbiol Infect Dis. 2014;33:919-26. http://dx.doi.org/10.1007/s10096-013-2027-1

5. Magobo RE, Corcoran C, Seetharam S, Govender NP. Candida auris-associated candidemia, South Africa. Emerg Infect Dis. 2014;20:1250-1. http://dx.doi.org/10.3201/eid2007.131765

6. Chakrabarti A, Sood P, Rudramurthy SM, Chen S, Kaur H, Capoor M, et al. Incidence, characteristics and outcome of ICUacquired candidemia in India. Intensive Care Med. 2015;41:28595. http://dx.doi.org/10.1007/s00134-014-3603-2

7. Emara M, Ahmad S, Khan Z, Joseph L, Al-Obaid I, Purohit P, et al. Candida auris candidemia in Kuwait, 2014. Emerg Infect Dis. 2015;21:1091-2. http://dx.doi.org/10.3201/eid2106.150270

8. Calvo B, Melo ASA, Perozo-Mena A, Hernandez M, Francisco EC, Hagen F, et al. First report of Candida auris in America: clinical and microbiological aspects of 18 episodes of candidemia. J Infect. 2016;73:369-74. http://dx.doi.org/10.1016/j.jinf.2016.07.008

9. Morales-López SE, Parra-Giraldo CM, Ceballos-Garzón A, Martínez HP, Rodríguez GJ, Álvarez-Moreno CA, et al. Invasive infections with multidrug-resistant yeast Candida auris, Colombia. Emerg Infect Dis. 2017;23:162-4. http://dx.doi.org/ 10.3201/eid2301.161497

10. Schelenz S, Hagen F, Rhodes JL, Abdolrasouli A, Chowdhary A, Hall A, et al. First hospital outbreak of the globally emerging 
Candida auris in a European hospital. Antimicrob Resist Infect Control. 2016;5:35. http://dx.doi.org/10.1186/s13756-016-0132-5

11. Vallabhaneni S, Kallen A, Tsay S, Chow N, Welsh R, Kerins J, et al.; MSD. Investigation of the first seven reported cases of Candida auris, a globally emerging invasive, multidrug-resistant fungus-United States, May 2013-August 2016. MMWR Morb Mortal Wkly Rep. 2016;65:1234-7. http://dx.doi.org/10.15585/ mmwr.mm6544e1

12. Ben-Ami R, Berman J, Novikov A, Bash E, Shachor-Meyouhas Y, Zakin S, et al. Multidrug-resistant Candida haemulonii and C. auris, Tel Aviv, Israel. Emerg Infect Dis. 2017;23:195-203. http://dx.doi.org/10.3201/eid2302.161486

13. Ruiz Gaitán AC, Moret A, López Hontangas JL, Molina JM, Aleixandre López AI, Cabezas AH, et al. Nosocomial fungemia by Candida auris: first four reported cases in continental Europe. Rev Iberoam Micol. 2017;34:23-7. http://dx.doi.org/10.1016/ j.riam.2016.11.002

14. Satoh K, Makimura K, Hasumi Y, Nishiyama Y, Uchida K, Yamaguchi H. Candida auris sp. nov., a novel ascomycetous yeast isolated from the external ear canal of an inpatient in a Japanese hospital. Microbiol Immunol. 2009;53:41-4. http://dx.doi.org/10.1111/j.1348-0421.2008.00083.x

15. Welsh RM, Bentz ML, Shams A, Houston H, Lyons A, Rose LJ, et al. Survival, persistence, and isolation of the emerging multidrug-resistant pathogenic yeast Candida auris on a plastic healthcare surface. J Clin Microbiol. 2017;55:2996-3005. http://dx.doi.org/10.1128/JCM.00921-17

16. Centers for Disease Control and Prevention. Recommendations for identification of Candida auris [cited 2017 Oct 18]. https://www.cdc.gov/fungal/diseases/candidiasis/ recommendations.html

17. Mizusawa M, Miller H, Green R, Lee R, Durante M, Perkins R, et al. Can multidrug-resistant Candida auris be reliably identified in clinical microbiology laboratories? J Clin Microbiol. 2017;55:638-40. http://dx.doi.org/10.1128/JCM.02202-16

18. Lockhart SR, Etienne KA, Vallabhaneni S, Farooqi J, Chowdhary A, Govender NP, et al. Simultaneous emergence of multidrug resistant Candida auris on 3 continents confirmed by whole genome sequencing and epidemiological analyses. Clin Infect Dis. 2017;64:134-40. http://dx.doi.org/10.1093/cid/ciw691

19. Centers for Disease Control and Prevention. Clinical alert to U.S. healthcare facilities - June 2016. Global emergence of invasive infections caused by the multidrug-resistant yeast Candida auris [cited 2017 Feb 28]. https://www.cdc.gov/fungal/diseases/ candidiasis/candida-auris-alert.html

20. New York State Department of Health. Health advisory: alert to New York State healthcare facilities regarding the global emergence of invasive infections caused by the multidrug-resistant yeast Candida auris [cited 2017 Nov 24]. https://www.health. ny.gov/diseases/communicable/c_auris/docs/2016-08_candida auris_advisory.pdf

21. New York State Department of Health. Health advisory: alert to New York State clinical laboratories: identification and reporting of suspected Candida auris isolates [cited 2017 Nov 24]. https://www.health.ny.gov/diseases/communicable/c_auris/docs/ 2016-11_candida_auris_advisory.pdf

22. Stevenson LG, Drake SK, Shea YR, Zelazny AM, Murray PR. Evaluation of matrix-assisted laser desorption ionization-time of flight mass spectrometry for identification of clinically important yeast species. J Clin Microbiol. 2010;48:3482-6. http://dx.doi.org/10.1128/JCM.00687-09

23. Clinical and Laboratory Standards Institute. M27-S4 reference method for broth dilution antifungal susceptibility testing of yeasts: fourth informational supplement. Wayne (PA): The Institute; 2012.

24. Leach L, Zhu Y, Chaturvedi S. Development and validation of a real-time PCR assay for rapid detection of Candida auris from surveillance samples. J Clin Microbiol. 2017;56:e01223-17. http://dx.doi.org/10.1128/JCM.01223-17

25. Tsay S, Welsh RM, Adams EH, Chow NA, Gade L, Berkow EL, et al. Notes from the field: ongoing transmission of Candida auris in health care facilities-United States, June 2016-May 2017. MMWR Morb Mortal Wkly Rep. 2017;66:514-5. http://dx.doi.org/ 10.15585/mmwr.mm6619a7

26. Okinda N, Kagotho E, Castanheira M, Njuguna A, Omuse G, Makau P, et al. Candidemia at a referral hospital in sub-Saharan Africa: emergence of Candida auris as a major pathogen. Poster presented at: European Congress of Clinical Microbiology and Infectious Diseases; 2014 May 10-13; Barcelona, Spain.

27. New York State Department of Health. Get the facts about Candida auris [cited 2017 Dec 28]. https://www.health.ny.gov/diseases/ communicable/c_auris/

Address for correspondence: Emily Lutterloh, New York State

Department of Health, Empire State Plaza, Corning Tower, Rm 523, Albany, NY 12237, USA; email: emily.lutterloh@health.ny.gov 